\title{
Agradecimento aos Avaliadores
}

O Corpo Editorial da Revista Neurociências agradece a todos os colegas que altruisticamente colaboraram com a avaliação dos artigos submetidos, revisando o conteúdo científico e técnico, fazendo revisóes abertas e realizando os Editoriais durante o ano de 2012.

Adriana Teresa Silva UNIVAS MG

Afonso C Neves UNIFESP SP

Alan Eckeli FMRP USP SP

Alba BS Fernandes UNIFESO RJ

Alberto Rasia-Filho UFCSPA RS

Aline M Toledo UFMS MS

Aline de Sousa Pagnussat UFCSPA RS

Amabile V Arias Unicamp SP

Ana Flávia Naoum de Almeida Unilago SP

Ana Lucia C Prado UFSM RS

Ana Maria Maaz Alvarez USP SP

Ana Paula Rocha Oliveira Unifran SP

Ana Paula Santos UFVJM MG

Anderson K Grzesiuk Univag MT

Andrea S Navarro IIEPAE, Uniban SP

Angela S M Salinet University of Leicester, UK

Anna Amélia P Motta Oishi UNIFESP SP

Anna Carolina X Chaves UNIFESP SP

Armando de Oliveira Neto Hosp C. Fontoura SP

Artemis S Nascimento Rodrigues Unifap AP

Caio Imaizumi FMABC SP

Camila Torriani-Pasin EEFE - USP SP

Carla da Silva Santana FMRP USP SP

Carlos B Mello Monteiro EACH USP SP

Carlos RM Rieder UFCSPA RS

Caroline Dani Universidade de Caxias do Sul RS

Cibelle KMR Formiga UEG GO

Clarisse Potasz UNIFESP SP

Clynton L Correa UFPR PR

christiane menegheti

Cristina SC de Sá UNIFESP Santos SP

Cristina Iwabe Unicamp SP

Cristina Y Toyoda UNESP SP

Dayane Nunes de Oliveira GO
Jadir Camargo Lemos UFSM RS

Jamary de Oliveira Filho UFBA BA

Janaina CP Neves USP SP

Janaine C Polese UPF RS

João Eduardo Araújo FMRP USP SP

Jocemar Ilha UDESC SC

Jocivana Oliveira da Silva UNIFAP AP

Joelma KSF Paschoal UNIFESP SP

Jose Fabio Leopoldino UFS SE

Jose Guilherme Mendes Pereira Caldas USP SP

Jose Olimpio T Sousa UFMG MG

José Renato de Oliveira Leite Unasp SP

Juliana SM Pasin Unifra RS

Karine Caldeira USP RP SP

Laiz HCT Guimarães UNILAVRAS MG

Lázaro J Teixeira UNIFESP Centro Cochrane

Leonardo Baldaçara UFT TO

Leticia Moraes de Aquino Unifesp SP

Luanda Andre Collange Uninove SP

Lucas R Nascimento UFMG MG

Luciane A Barreto UNIFESP SP

Luis Guilherme GC Guerra Conscientia NECSM SP

Luis Henrique Telles da Rosa UFCSPA RS

Magali TQ Grave UNIVATES RS

Marcela Silagi USP SP

Marcia Midori Morimoto USCS SP

Marco AF Alves UNIMES UNISANTA SP

Marco Antonio Orsini Neves UFF RJ

Marcus VM Gonçalves Joinville SC

Marcos RG Freitas UFRJ RJ

Maria da Graça N Mazzacoratti UNIFESP SP

Maria Emília FA Melo UPE PE

Maria Martha Bernardi UniABC, UNIP SP

Marilia R Callegari UNIFESP SP 
Debora AG Alves Unilavras MG

Debora Amado Scerni Unifesp SP

Debora Aparecida Lentini-Oliveira Unifesp SP

Denis B Bichuetti UNIFESP SP

Deusvenir de Souza Carvalho Unifesp SP

Dionísia Aparecida Cusin Lamônica FOB USP SP

Douglas Martins Braga UNIFESP SP

Ébe dos Santos Monteiro UNIFESP SP

Eddy Krueger UTFPR PR

Edgard Morya UNIFESP SP

Eduardo NP Cillo PUC Minas MG

Edmar Zanotelli UNIFESP SP

Elaine Maria Silva Barbosa Juiz de Fora MG

Eliane MF Carvalho UNIFESP SP

Eliane Araujo Oliveira UFPB PB

Enio WA Cacho Unicamp SP

Fabiola P Rebouças Santa Casa SP

Fabricio R Araujo FCMSCSP SP

Felipe Stigger - ULBRA Santa Maria RS

Fernando Cesar I Marcucci UEL - Londrina PR

Fernando R Carvalho Unifesp SP

Flavia Doná Uniban SP

Francis Meire Favero UNIFESP / FIBRA SP

Francisco EC Cardoso UFMG MG

Geraldo NV Rizzo Hosp. Moinho de Ventos RS

Giedre Felix USP Bauru SP

Gilberto Mastrocola Manzano UNIFESP SP

Gisele Sampaio Silva UNIFESP SP

Glenda CB Lacerda UFF RJ

Gustavo Christofoletti UFMS MS

Gustavo de Val Barreto FEAD MG

Halina Cidrini Ferreira UFRJ RJ

Heidi Sanders UFMRP USP SP

Helio AG Teive UFPR PR

Iscia T Lopes Cendes Unicamp SP

Izabel Baraldi Unimep SP

Jacqueline MRS Leite UNILAVRAS MG
Marina Brito UNIFESP SP

Marisa Fukuda FMRP USP SP

Max D Pereira Unifesp SP

Osório Abath Neto

Osvaldo Pelozo Júnior CUni FIEO Osasco SP

Osvladir Custódio UNIFESP SP

Paulo Abe Unilavras MG

Paulo HP Bertolucci UNIFESP SP

Raquel Faria USP RP SP

Raquel R Brito UFMG MG

Regiane Luz Carvalho UNIFAE SP

Reginaldo C Silva Filho UNIFESP SP

Rivelilson M Freitas UFPI PI

Roberto CP Prado UFSE SE

Rodrigo C Schuster FSG RS

Rodrigo S Gomez Belo Horizonte MG

Rubens Gagliardi Santa Casa SP

Sara RM Almeida Unicamp SP

Sheila Gemelli de Oliveira UPF RS

Silvia Lanziotti Azevedo da Silva UFMG MG

Sonia MD Brucki FMUSP UNIFESP SP

Tabajara O Gonzales Uninove SP

Tatiana AB Bressel UFRS RS

Thaís Branquinho Oliveira Fragelli UNB DF

Thais Ioshimoto UNIFESP SP

Taiza EG Santos-Pontelli FMRP-USP SP

Thatiane M Campos-Zanelli Unicamp SP

Tiago S Carvalho FEEVALE - RS

Valeria Meireles Carril Elui FMUSP RP SP

Valeria Neves Kroeff Mayer UNISC SC

Valeria Sella UNIFESP SP

Vanessa R Ferreira Unifesp SP

Vicente Leitão ICNE CE

Victor H Bastos UFVJM MG

Vitor Engrácia Valenti UNIFESP SP

Ylmar Correa Neto UFSC SC 